FACTA UNIVERSITATIS (NIŠ)

Ser. Math. Inform. Vol. 36, No 2 (2021), 409-417

https://doi.org/10.22190/FUMI200916030A

Original Scientific Paper

\title{
THE STATISTICAL MULTIPLICATIVE ORDER CONVERGENCE IN RIESZ ALGEBRAS
}

\author{
Abdullah Aydın \\ Department of Mathematics, Muş Alparslan University \\ 49250 Muş, Turkey
}

\begin{abstract}
The statistically multiplicative convergence in Riesz algebras was studied and investigated with respect to the solid topology. In the present paper, the statistical convergence with the multiplication in Riesz algebras is introduced by developing topology-free techniques using the order convergence in vector lattices. Moreover, we give some relations with the other kinds of convergences such as the order statistical convergence, the mo-convergence, and the order convergence.

Key words: Statistical convergence, Statistical mo-convergence, Order convergence, Order statical convergence, Riesz algebra, Riesz spaces, $f$-algebra
\end{abstract}

\section{Introduction and Preliminaries}

Steinhaus introduced the concept of statistical convergence in [15] that is a generalization of the convergence of real sequences. Another important concept of functional analysis is vector lattice (or, Riesz spaces) which was introduced by F. Riesz [13]. We refer the reader for applications of Riesz spaces to [1, 2, 3, 4, 5, 18]. We aim to combine concepts of the order and the statistical convergence, and the multiplicative on Riesz algebras, and so, we introduce the convergence on Riesz algebras without topological structure.

For the statistical convergence, the natural density of subsets of $\mathbb{N}$ has critical points. Take a subset $B$ in $\mathbb{N}$. Then the unique limit $\lim _{n \rightarrow \infty} \frac{1}{n}|\{k \leq n: k \in A\}|$ is

Received September 16, 2020; accepted March 26, 2021

Communicated by Božidar Popović

Corresponding Author: Abdullah Aydın, Department of Mathematics, Muş Alparslan University, 49250 Muş, Turkey | E-mail: aaydin.aabdullah@gmail.com

2010 Mathematics Subject Classification. Primary 40A05; Secondary 46B42, 46J40, 47B65

(C) 2021 By University of Niš, Serbia | Creative Commons License: CC BY-NC-ND 
said to be the natural density of $B$ whenever it exists. Also, we abbreviate it as $\delta(A)$. Now, take a sequence $\left(x_{n}\right)$ of reel numbers. If, for a given $\varepsilon>0$, the limit

$$
\lim _{n \rightarrow \infty} \frac{1}{n}\left|\left\{k: n \geq k,\left|x_{n}-x\right|>\varepsilon\right\}\right|=0 .
$$

exists then it is called that $\left(x_{n}\right)$ statistical converges to $x$. Several applications and generalizations about the statistical convergence have been investigated by several authors (cf. $[3,7,8,11,16,17])$. In this paper, we abbreviate the cardinality of subsets in the vertical bar.

Let " $\leq$ " be an order relation on a reel vector space $E$. Then $E$ is called ordered vector space if $\beta x \leq \beta y$ and $x+z \leq y+z$ hold in $E$ for all $\beta \in \mathbb{R}_{+}$and $z \in E$ whenever $x \leq y$. Let $E$ be an order vector space. Then it is said to be vector lattice or Riesz space if, for every pair $x, y \in E$, we have

$$
x \vee y=\sup \{x, y\} \text { and } x \wedge y=\inf \{x, y\}
$$

in $E$. Moreover, a Riesz space is called $\sigma$-order or $\sigma$-Dedekind complete whenever each countable and bounded above subset has a supremum. Take an element $x$ in a vector lattice $E$. Then $x^{+}:=x \vee 0$ is the positive part, $x^{-}:=(-x) \vee 0$ is the negative part, and $|x|:=x \vee(-x)$ is the module of $x$. So, in this paper, we use the vertical bar $|\cdot|$ of elements for the module of the given elements. Some works on Riesz spaces with statistical convergence have done. For example, a characterization of statistical convergence was introduced by Ercan in [8], and Aydin introduced the statistical convergence with unbounded order convergence [3]. The crucial point in Riesz spaces is the order convergence. Thus, we continue with its definition.

Definition 1.1. The order convergence of a sequence $\left(x_{n}\right)$ to an element $x$ in a Riesz space $E$ defined as follows:

(i) There exists another sequence $\left(y_{n}\right)$ in $E$ such that inf $y_{n}=0$ and $y_{n} \downarrow$ in $E$ (i.e., $\left.y_{n} \downarrow 0\right)$;

(ii) $\left|x_{n}-x\right| \leqslant y_{n}$ for each $n \in \mathbb{N}$.

Next, we turn our attention to Riesz algebras. If, for an associative algebraic vector lattice $E, x \cdot y \in E_{+}$holds for every $x, y \in E_{+}$then $E$ is called a Riesz algebra (or, shortly, l-algebra). Also, if $x \cdot y=y \cdot x$ holds for all pair $x, y \in E$ then $E$ is said to be commutative. For much more information on $l$-algebras, we refer $[2,6,10,12,18]$. Aydın and Et introduced the statistical convergence on Riesz algebra with the solid topology [7].

Definition 1.2. Let $E$ be a Riesz algebra. Then it is called

(1) $d$-algebra if $(x \wedge y) \cdot u=(x \cdot u) \wedge(y \cdot u)$ and $u \cdot(x \wedge y)=(u \cdot x) \wedge(u \cdot y)$ hold for each $x, y \in E$ and $u \in E_{+}$; 
(2) unital if $E$ has a multiplicative unit.

(3) $f$-algebra whenever we have $y \wedge(u \cdot x)=0$ and $y \wedge(x \cdot u)=0$ for all $y \wedge x=0$, $x, y \in E$ and $u \in E_{+}$.

It is clear that $u \cdot y \leq u \cdot x$ holds in Riesz algebras for elements $y \leq x$ and for all positive vector $u$. Remind that if $\frac{1}{n} x \downarrow 0$ holds for any positive vector $x$ in a Riesz space $E$ then it is said to be Archimedean Riesz space. By considering [18, Thm.140.10], one can see that each Archimedean $f$-algebra has the commutative property. In the works (cf. $[4,5,6,10,12]$ ), the reader can find more features and some kinds of convergences in $l$-algebras.

Example 1.1. Consider the set of orthomorphisms on a Riesz space $E$

$$
\operatorname{Orth}(E):=\left\{\pi \in L_{b}(E): x \perp y \text { implies } \pi x \perp y\right\} .
$$

That is, $|\pi x| \wedge|y|=0$ whenever $|x| \wedge|y|=0$ in $E$. Now, let's take $E$ as an $\sigma$-Dedekind complete Riesz space. Then, by using [12, Thm.15.4], we have $\operatorname{Orth}(E)$ is an $\sigma$-Dedekind complete, and also, $\operatorname{Orth}(E)$ is an unital $f$-algebra.

For much more examples of Riesz algebras see for example [6, 10, 12]. In this paper, unless otherwise, we assume that all Riesz spaces are Archimedean and all multiplications are commutative.

\section{The statistical mo-convergence}

We define the statistical convergence in Riesz algebras with respect to multiplicative order convergence in this section. To give this notion, we use the statistical monotonicity for real sequences that was introduced by Salat in [14]. We take the following notions from [4] and [16].

\section{Definition 2.1.}

(a) Let $\left(x_{n}\right)$ be a sequence in a Riesz algebra $E$. Then it is called multiplicative order convergent to $x \in E$ whenever $u \cdot\left|x_{\alpha}-x\right| \stackrel{\circ}{\rightarrow} 0$ for every $u \in E_{+}$. Abbreviated as $x_{\alpha} \stackrel{\mathrm{mo}}{\longrightarrow} x$.

(b) Let $\left(q_{n}\right)$ be a sequence in a Riesz space $E$. Then it is called statistical monotone convergent to $x \in E$ if there exists a subset $J$ in $\mathbb{N}$ with $\delta(J)=1$ and $\left(q_{n_{k}}\right)_{k} \downarrow x$. It is abbreviated as $q_{n} \downarrow^{s t} x$.

(c) A sequence $\left(x_{n}\right)$ is said to be statistical order converges to $x$ in a vector lattice $E$ if there are a subset $\delta(J)=1$ and a sequence $y_{n} \downarrow^{\text {st }} 0$ with $\left|x_{n}-x\right| \leq y_{n}$ for all $n \in J$.

We give a basic observation in the following result.

Lemma 2.1. Every order convergent monotone sequence is statistical monotone convergent in vector lattices. 
Proof. Take an order convergent sequence $x_{n} \stackrel{\circ}{\rightarrow} x$ in a Riesz space $E$ such that $x_{n} \downarrow$ (i.e., $x_{n} \downarrow x$ ). Now, we can choose $J$ in Definition 2.1(b) as $\mathbb{N}$. Then we have $\delta(J)=1$ and $x_{n} \downarrow x$ on $J$. So, we obtain the desired, $x_{n} \downarrow^{\text {st }} x$, result.

Now, motivated from above definitions, we give the following crucial notion.

Definition 2.2. Let $E$ be an $l$-algebra and $\left(x_{n}\right)$ be a sequence in $E$. Then $\left(x_{n}\right)$ is called statistical multiplicative order convergent (or, statistical mo-convergent, shortly) to $x \in E$ if, for each positive element $u \in E_{+}$, there exists a subset $J$ of the natural numbers with $\delta(J)=1$ and a sequence $q_{n} \downarrow^{\text {st }} 0$ such that

$$
\left|x_{n_{j}}-x\right| \cdot u \leqslant q_{n_{j}}
$$

for all $n_{j} \in J$. We abbreviate it as $x_{n} \stackrel{\text { st-mo }}{\longrightarrow} x$.

It can be seen that $x_{n} \stackrel{\text { st-mo }}{\longrightarrow} x$ if, for each $u \in E_{+}$, there exists a sequence $q_{n} \downarrow^{\text {st }} 0$ such that the natural density of the set $\left\{n \in \mathbb{N}:\left|x_{n}-x\right| \cdot u \not \leq q_{n}\right\}$ is equal to zero.

Proposition 2.1. The mo-convergence implies the statistical mo-convergence in l-algebras.

Proof. Assume that a sequence $\left(x_{n}\right)$ is mo-convergent to $x$ in an $l$-algebra $E$. Let's fix $u \in E_{+}$. Then, following from Definition 2.1(a), we have $\left|x_{n}-x\right| \cdot u \stackrel{\circ}{\rightarrow} 0$. Thus, there is a sequence $y_{n} \downarrow 0$ in $E$ such that $\left|x_{n}-x\right| \cdot u \leqslant y_{n}$ holds for all $n \in \mathbb{N}$. So, by applying Lemma 2.1, we obtain $y_{n} \downarrow^{\text {st }} 0$. Since $u \in E_{+}$is arbitrary, if we take the subset $J$ as $\mathbb{N}$ then we get the desired result, $x_{n} \stackrel{\text { st-mo }}{\longrightarrow} x$.

It is known that the order convergence does not imply the mo-convergence in $l$-algebras because $l$-algebras do not have the infinite distributive property, i.e., if $\inf (A)$ exists and positive for any subset $A$ of an $l$-algebra $E$ then the infimum of the subset $u \cdot A$ exists and $\inf (u \cdot A)=u \cdot \inf (A)$ for every $u \in E_{+}$(see, [4, p.2] and [6, Thm.12]). By the way, the order and the statistical order convergences do not imply the statistical mo-convergent, in general. But, we have a positive implication in the following work.

Theorem 2.1. If $\left(x_{n}\right)$ in a d-algebra is order or statistical order convergent sequence then it is statistical mo-convergent to their order or statistical order limit points.

Proof. Assume that $\left(x_{n}\right)$ statistical order converges to $x$ in a $d$-algebra $E$. We show that $\left(x_{n}\right)$ is statistical mo-convergent to $x$. Similarly, one can show the other case. Following from Definition 2.1(c), there exists a sequence $q_{n} \downarrow^{\text {st }} 0$ and a subset $J$ of the natural numbers with $\delta(J)=1$ such that $\left|x_{n_{j}}-x\right| \leqslant q_{n_{j}}$ for all $n_{j} \in J$. On the other hand, there is a subset $\delta(K)=1$, and also, $\left(q_{n_{k}}\right)$ is decreasing to zero 
because of $q_{n} \downarrow^{\text {st }} 0$. Next, consider the set $M=J \cap K$. Hence, following from the inequality $\delta(J)+\delta(K) \leq 1+\delta(J \cap K)$, we have $\delta(M)=1$. As a result, we obtain that $\left|x_{n_{m}}-x\right| \leqslant q_{n_{m}} \downarrow 0$. Therefore, we get $\left|x_{n_{m}}-x\right| \cdot u \leqslant\left(q_{n_{m}} \cdot u\right) \downarrow 0$ for all $u \in E_{+}$because every $d$-algebra having infinite distributive properties; see [6, Thm.12.]. Thus, for every $u \in E_{+}$, we can obtain a sequence $w_{n}=\left(q_{n} \cdot u\right) \downarrow^{\text {st }} 0$, and also, $\left|x_{n}-x\right| \cdot u \leqslant w_{n}$ holds on $M$, i.e., we get $x_{n} \stackrel{\text { st-mo }}{\longrightarrow} x$.

In the following result, we give a partial answer for the converse implication of Theorem 2.1

Proposition 2.2. Every statistical mo-convergent sequence in an unital $f$-algebra is statistical order convergent to its statistical mo-limit.

Proof. Let $\left(x_{n}\right)$ be a statistical mo-convergent sequence in an unital $f$-algebra $E$ with the multiplicative unit $e$. Then there exists a sequence $q_{n} \downarrow^{\text {st }} 0$ such that the natural density of the subset $\left\{n \in \mathbb{N}:\left|x_{n}-x\right| \cdot u \not \leq y_{n}, \forall u \in E_{+}\right\}$is equal to zero. By applying [18, Thm.142.1(v)], in view of $e=e \cdot e=e^{2} \geq 0$, one clearly can obtain that unit element is positive in $E$. Thus, in a special case, we can take $u=e \in E_{+}$. Then we have

$$
\delta\left(\left\{n:\left|x_{n}-x\right| \not \leq y_{n}\right\}\right)=\delta\left(\left\{n:\left|x_{n}-x\right| \cdot e \not \leq y_{n}\right\}\right)=0 .
$$

Therefore, we obtain that $\left(x_{n}\right)$ statistical order converges to $x$.

\section{Main Results of the Statistical mo-Convergence}

In this section, we give the main results and properties of the statistical moconvergence. First of all, to mention the uniqueness of the statistical mo-limit, we need the notion of semiprime $l$-algebra. Consider an element $x$ in a Riesz algebra $E$ with $x^{n}=0$ for some natural numbers $n \in \mathbb{N}$ then it is said to be a nilpotent element. Moreover, if the only nilpotent element of a Riesz algebra $E$ is zero element then $E$ is called semiprime (cf., $[9,10,12,18]$ ).

Lemma 3.1. Let $\left(x_{n}\right)$ be a sequence of nilpotent elements of an $f$-algebra E. If $x_{n} \stackrel{\text { st-mo }}{\longrightarrow} x$ then $x$ is a nilpotent element of $E$.

Proof. Suppose $x_{n} \stackrel{\text { st-mo }}{\longrightarrow} x$. Fix a positive element $u \in E_{+}$. Then there exists a sequence $q_{n} \downarrow^{s t} 0$ and a subset $\delta(J)=1$ such that $\left|x_{n_{j}}-x\right| \cdot u \leqslant q_{n_{j}}$ for all $n_{j} \in J$. Now, following from [12, Prop.10.2(iii)] and [18, Thm.142.1(ii)], we have

$$
q_{n_{j}} \geq\left|x_{n_{j}}-x\right| \cdot u=\left|x_{n_{j}} \cdot u-x \cdot u\right|=|x \cdot u|
$$

because $\left(x_{n}\right)$ consists of nilpotent elements. Thus, we obtain $|x \cdot u|=0$, i.e., we have $x \cdot u=0$ for every $u \in X_{+}$because of $q_{n_{j}} \downarrow 0$. Then $x \cdot y=0$ for each $y \in E$ because of $y=y^{+}-y^{-}$and $y^{+}, y^{-} \in E_{+}$. Therefore by using [9, p.157], one can see that $x$ is also a nilpotent element. 
Proposition 3.1. The limit of a statistically mo-convergent sequence is uniquely determined in semiprime $f$-algebras.

Proof. Suppose that $\left(x_{n}\right)$ is a statistically mo-convergent to $x$ and $y$ sequence in a semiprime $f$-algebra $E$. Fix $u \in E_{+}$. Then there exists sequences $q_{n} \downarrow^{s t} 0$ and $p_{n} \downarrow^{\text {st }} 0$, and subsets $J$ and $K$ of the natural numbers with $\delta(J)=\delta(K)=1$ such that $\left|x_{n_{j}}-x\right| \cdot u \leqslant q_{n_{j}}$ and $\left|x_{n_{k}}-y\right| \cdot u \leqslant p_{n_{k}}$ for all $n_{j} \in J$ and $n_{k} \in K$. Choose $M=J \cap K$. Thus, we have $\delta(M)=1,\left|x_{n_{m}}-x\right| \cdot u \leqslant q_{n_{m}}$ and $\left|x_{n_{m}}-y\right| \cdot u \leqslant p_{n_{m}}$ for every $n_{m} \in M$. Now, it follows that

$$
|x-y| \cdot u \leq\left|x_{n_{m}}-x\right| \cdot u+\left|x_{n_{m}}-y\right| \cdot u
$$

satisfies for every $m \in \mathbb{N}$. Thus, we obtain $|x-y| \cdot u=0$. Since $u$ is arbitrary, one can see that $|x-y|$ is a nilpotent element in $E$ (cf. [9, p.157]). Therefore, we get $|x-y|=0$, i.e., we have $x=y$ because of $E$ is semiprime.

Next, we give several results that are parallel to some kinds of statistical convergence such as [3, Thm.2.2.] and [1, Thm.2.17.].

Theorem 3.1. If $x_{n} \stackrel{\text { st-mo }}{\longrightarrow} x$ and $y_{n} \stackrel{\text { st-mo }}{\longrightarrow} y$ in an l-algebra $E$ then the following holds:

(i) The lattice operations are statistical mo-order continuous;

(ii) $x_{n} \stackrel{\text { st-mo }}{\longrightarrow} x$ iff $\left(x_{n}-x\right) \stackrel{\text { st-mo }}{\longrightarrow} 0$ iff $\left|x_{n}-x\right| \stackrel{\text { st-mo }}{\longrightarrow} 0$;

(iii) The statistical mo-limit is linear;

(iv) $x_{n_{k}} \stackrel{\text { st-mo }}{\longrightarrow} x$ for any subsequence $\left(x_{n_{k}}\right)$ of $\left(x_{n}\right)$;

(v) $E_{+}$that is the positive cone of $E$ is closed under the statistical mo-convergence whenever $E$ is semiprime $f$-algebra.

Proof. (i) It is enough to show that $\left(x_{n} \vee y_{n}\right)$ statistical mo-converges to $x \vee y$. Take fixed $u \in E_{+}$. Since $x_{n} \stackrel{\text { st-mo }}{\longrightarrow} x$ and $y_{n} \stackrel{\text { st-mo }}{\longrightarrow} y$, by the same argument in the proof of Proposition 3.1, there exists a subset of the natural numbers with $\delta(M)=1$ and sequences $q_{n} \downarrow^{s t} 0$ and $p_{n} \downarrow^{s t} 0$ such that $\left|x_{n_{m}}-x\right| \cdot u \leqslant q_{n_{m}}$ and $\left|x_{n_{m}}-y\right| \cdot u \leqslant p_{n_{m}}$ for every $n_{m} \in M$. By using [2, Thm.1.2(2)], we have

$$
\left|x_{n_{m}} \vee y_{n_{m}}-x \vee y\right| \cdot u \leq\left|x_{n_{m}}-x\right| \cdot u+\left|y_{n_{m}}-y\right| \cdot u \leq q_{n_{m}}+p_{n_{m}}
$$

for each $m \in \mathbb{N}$. Hence, if we denote a sequence $r_{n}:=q_{n}+p_{n}$ then we have $\left|x_{n_{m}} \vee y_{n_{m}}-x \vee y\right| \cdot u \leq r_{n_{m}}$ and $r_{n} \downarrow^{\text {st }} 0$. Hence, we obtain $x_{n} \vee y_{n} \stackrel{\text { st-mo }}{\longrightarrow} x \vee y$ in E.

One can get $(i i)$ and $(i v)$ directly from the definition of the statistical moconvergence. Also, $($ iii $)$ is similar to $(i)$. 
(v) Suppose that $\left(x_{n}\right)$ is non-negative and statistical mo-converges to $x \in E$. It follows from $(i)$ that $x_{n}=x_{n}^{+} \stackrel{\text { st-mo }}{\longrightarrow} x^{+}$, and also, following from Proposition 3.1, we obtain $x=x^{+}$. So, we get the desired, $x \in E_{+}$, result.

In the following result, we give a positive answer for the converse of Theorem 2.1.

Proposition 3.2. Ever monotone statistical mo-convergent sequence in a semiprime $f$-algebra order converges to its statistical mo-limit.

Proof. Suppose that a sequence $\left(x_{n}\right)$ in a semiprime $f$-algebra $E$ is increasing and statistical mo-convergent to $x \in E$. It is enough to show $x_{n} \uparrow x$. Let's fix an index $n_{0}$. It is clear that $x_{n}-x_{n_{0}} \in X_{+}$for each $n \geqslant n_{0}$. Now, by using linearity of statistical mo-limit, we have $x_{n}-x_{n_{0}} \stackrel{\text { st-mo }}{\longrightarrow} x-x_{n_{0}}$. Since $x_{n}-x_{n_{0}} \in E_{+}$, by applying Theorem 3.1(v), we can obtain $x-x_{n_{0}} \in E_{+}$, i.e., $x \geq x_{n_{0}}$. Thus, $x$ is an upper bound of $\left(x_{n}\right)$ because $x_{n_{0}}$ is arbitrary. Take another upper bound $y$ of $\left(x_{n}\right)$, i.e., $y \geq x_{n}$ for all $n$. Then we obtain $y-x_{n} \stackrel{\text { st-mo }}{\longrightarrow} y-x \in E_{+}$, or equivalently, we get $y \geqslant x$. Thus, $x_{n} \uparrow x$.

Proposition 3.3. If $0 \leq y_{n} \leq x_{n}$ holds for every natural number $n \in \mathbb{N}$ and $x_{n} \stackrel{\text { st-mo }}{\longrightarrow} 0$ in an l-algebra $E$ then we have $y_{n} \stackrel{\text { st-mo }}{\longrightarrow} 0$ in $E$.

Proof. Fix $u \in E_{+}$. Since $x_{n} \stackrel{\text { st-mo }}{\longrightarrow} 0$, there exist a subset $\delta(J)=1$ and a sequence $q_{n} \downarrow^{\text {st }} 0$ such that $x_{n_{j}} \cdot u \leqslant q_{n_{j}}$ for every $n_{j} \in J$. So, we have $0 \leq y_{n_{j}} \leq x_{n_{j}}$, and so, following from the inequality $y_{n_{j}} \cdot u \leq x_{n_{j}} \cdot u$ for all $j$, we obtain the desired, $y_{n} \stackrel{\text { st-mo }}{\longrightarrow} 0$, result.

Recall that every order convergent sequence in a $d$-algebra is statistical $m o$-convergent (see, Theorem 2.1). But, for the general case, we give the following notions.

Definition 3.1. Assume $\left(x_{n}\right)$ is a sequence in a Riesz algebra $E$. Then

(a) $\left(x_{n}\right)$ in $E$ is called statistical mo-Cauchy whenever the sequence $\left(x_{n}-x_{m}\right)_{(m, n) \in \mathbb{N} \times \mathbb{N}}$ statistical mo-converges to 0 ;

(b) $E$ is said to be statistical mo-complete whenever each statistical mo-Cauchy sequence is statistical mo-convergent;

(c) E is called statistical mo-continuous whenever every order convergent sequence is statistical mo-convergent.

Proposition 3.4. The following statements are equivalent for arbitrary Riesz algebra E.

(i) E is statistical mo-continuous; 
(ii) $x_{n} \downarrow 0$ in $X$ implies $x_{n} \stackrel{\text { st-mo }}{\longrightarrow} 0$.

Proof. We show the implication $(i i) \Rightarrow(i)$ because the converse is trivial. Take a sequence $x_{n} \stackrel{\circ}{\rightarrow} x$ in $E$. Thus, there exists a sequence $y_{n} \downarrow 0$ in $E$ such that $\left|x_{n}-x\right| \leq$ $y_{n}$ for every $n \in \mathbb{N}$. Moreover, by using (ii), we have $y_{n} \stackrel{\text { st-mo }}{\longrightarrow} 0$ because of $y_{n} \downarrow 0$. So, it follows from Proposition 3.3 that $\left|x_{\alpha}-x\right|$ is also statistical mo-converges to zero. Therefore, by considering Theorem $3.1($ ii $)$, we have $x_{n} \stackrel{\text { st-mo }}{\longrightarrow} x$.

Theorem 3.2. Let $E$ be a statistical mo-continuous and mo-complete semiprime $f$-algebra. Then $E$ is $\sigma$-order complete.

Proof. Consider a sequence $0 \leq x_{n} \uparrow \leq x$ in $E$. Thus, by considering [1, Lem.1.39.], it is enough to show the existence of $\sup x_{n}$. Now, by [2, Lem.4.8.], we have a new sequence $\left(y_{n}\right)$ in $E$ with $\left(y_{n}-x_{n}\right) \downarrow 0$. Then it follows from Proposition 3.4 that $\left(y_{n}-x_{n}\right) \stackrel{\text { st-mo }}{\longrightarrow} 0$ because $E$ is statistical mo-continuous. Next, by considering the linearity of statistical mo-limit, Proposition 3.3 and the following inequality

$$
\left|x_{n}-x_{m}\right| \leq\left|x_{n}-y_{n}\right|+\left|y_{n}-x_{m}\right|,
$$

we obtain that the sequence $\left(x_{n}\right)$ is a statistical mo-Cauchy. Thus, there is some $x \in E$ such that $x_{n} \stackrel{\text { st-mo }}{\longrightarrow} x$ because $E$ is statistical mo-complete. Now, by applying Proposition 3.2, since we have $x_{n} \uparrow x$, we obtain the $\sigma$-order completeness of $E$.

Proposition 3.5. If every increasing order bounded sequence in a semiprime $f$ algebra $E$ is statistical mo-convergent then $E$ is statistical mo-continuous.

Proof. Suppose $x_{n} \downarrow 0$. So, we show that it is statistical mo-convergent to 0 . Let's fix an index $n_{0}$ and consider a sequence $y_{n}:=x_{n_{0}}-x_{n}$ for $n \geqslant n_{0}$. It is clear that $0 \leq y_{n} \uparrow \leqslant x_{n_{0}}$. Therefore, we see that $\left(y_{n}\right)$ is increasing and order bounded sequence. Thus, by our assumption, one can say that $\left(y_{n}\right)$ is statistical moconvergent to some $y \in E$. Since $\left(y_{n}\right)$ is increasing and statistical mo-convergent, Proposition 3.2 gives the following equality

$$
y=\sup _{n \geqslant n_{0}} y_{n}=\sup _{n \geqslant n_{0}}\left(x_{n_{0}}-x_{n}\right)=x_{n_{0}} \text {. }
$$

Therefore, we have $y_{n}=x_{n_{0}}-x_{n} \stackrel{\text { st-mo }}{\longrightarrow} x_{n_{0}}$, or $x_{n} \stackrel{\text { st-mo }}{\longrightarrow} 0$. So by Proposition 3.4 , $E$ is statistical mo-continuous.

\section{REFEREN C E S}

1. C. D. Aliprantis and O. Burkinshaw: Locally Solid Riesz Spaces with Applications to Economics. American Mathematical Society, 2003. 
2. C. D. Aliprantis and O. Burkinshaw: Positive Operators. Springer, Dordrecht, 2006.

3. A. Aydin: The statistically unbounded $\tau$-convergence on locally solid Riesz spaces. Turk. J. Math. 44 (2020), 949-956.

4. A. Aydin: Multiplicative order convergence in f-algebras. Hacet. J. Math. Stat. 49 (2020), 998-1005.

5. A. Aydin: The multiplicative norm convergence in normed Riesz algebras. Hacet. J. Math. Stat. 50 (2021), 24-32.

6. A. Aydin, E. Emel'yanov and S. G. Gorokhova: Full lattice convergence on Riesz spaces. Indagat. Math. (in press) (2021). doi.org/10.1016/j.indag.2021.01.008

7. A. Aydin and M. ET: Statistically multiplicative convergence on locally solid Riesz algebras. Turk. J. Math. (in press) (2021).

8. Z. ERCAN: A characterization of u-uniformly completeness of Riesz spaces in terms of statistical u-uniformly pre-completeness. Demon. Math. 42 (2009), 383-387.

9. C. B. Huissmans: Lattice-Ordered Algebras and f-Algebras: a survey. Springer, Berlin, 1991.

10. C. B. Huijsmans and B. D. Pagter: Ideal theory in f-algebras. Trans. Amer. Math. Soc. 269 (1982), 225-245.

11. I. J. MAdDox: Statistical convergence in a locally convex space. Math. Proc. Cambr. Phil. Soc. 104 (1988), 141-145.

12. B. D. PAGTER: $f$-Algebras and Orthomorphisms. Ph. D. Thesis, University of California, Leiden, 1981.

13. F. RIEsz: Sur la décomposition des opérations fonctionelles linéaires. Atti D. Congr. Inter. D. Math., Bologna, 1928.

14. T. SAlat: On statistically convergent sequences of real numbers. Math. Slov. 30 (1980), 139-150.

15. H. Steinhaus: Sur la convergence ordinaire et la convergence asymptotique. Colloq. Math. 2 (1951), 73-74.

16. C. ŞEnCIMen and S. Pehlivan: Statistical order convergence in Riesz spaces. Math. Slov. 62 (2012), 557-570.

17. F. Temizsu and M. Eт: On statistically Köthe-Toeplitz duals. J. Math. Ineq. 13 (2019), 1147-1157.

18. A. C. ZaAnen: Riesz Spaces II. North-Holland Publishing Co., Amsterdam, 1983. 